\title{
Wireless sensing network transmission system with improved constant modulus algorithm
}

\author{
Wen-Tsai Sung
}

\begin{abstract}
To solve the instant signal convergence of wireless sensing network transmission system, this study proposes an improved constant modulus algorithm. The improved constant modulus algorithm can quickly find the convergent direction by adding error functions and learning rates based on the reserved original cost functions. After processed by a matched filter, the received consecutive time signal can be sampled in accordance with symbol interval or fraction interval, so that signal channels can be estimated or balanced. At last, this study verifies the raised method with quadrature amplitude modulation on wireless sensing network transmission system and improved constant modulus algorithm experiments which show that the learning rates and the performances of balancers are improved.
\end{abstract}

Keywords: Wireless sensor network, Transmission system, Constant modulus algorithm, 4QAM

\section{Introduction}

Signal channel balance is one of the fundamental issues of digital communication technology. The purpose is to overcome interferences among transmitted symbols (ISIs). These interferences are caused by the non-ideal characters of signal channels. When the baud rate is higher than $4,800 \mathrm{bit} / \mathrm{s}$ in the wireless sensing network transmission system and higher than $100 \mathrm{bit} / \mathrm{s}$ in the short-wave transmission system, a balancer is required. The communication channels may be unknown or changed. Thus, it is required to utilize training sequences to self-adaptively adjust balancers to remove the interferences among symbols. R.W. Lucky raised the earliest self-adaptive balancer and the balancing algorithm of zero forcing. Meanwhile, the minimum mean square error algorithm raised by Widrow and Hoff is used on signal channel balance and widely applied $[1,2]$.

For most digital communication systems, the characters of signal channel are usually unknown and varied along with time. Thus, to design a correspondent selfadaptive balancer, it is usually required to include the known training sequences in the data frame at the sending end to be transmitted to the receiving end. The

\footnotetext{
Correspondence: songchen@ncut.edu.tw
}

Department of Electrical Engineering, National Chin-Yi University of

Technology, No.57, Sec. 2, Zhongshan Rd.,Taiping Dist., Taichung 41170, Taiwan purpose is to conduct initial adjustments to the parameters in the balancer in order to guarantee quick convergence in a wide range. However, the balancer based on the training sequences will increase transmission costs and reduce the efficiency of the communication system. For example, in the GSM system, there are 25 symbols for training every 124 symbols. This causes $24 \%$ capacity loss. In high-frequency communication systems, due to the serious impacts of the multiple paths and attenuations of the ionosphere, the time used to transmit training signals may occupy $48 \%$ of total transmission time $[3,4]$.

In some important communication applications, it is required to realize balance without the assistance of training signals, so-called unknown balance. For example, in the communication of broadcasting or single-point-to-multiple-point communication, digital high-definition television (HDTV) is a typical example of broadcasting communication. Digital microwave link has serious attenuations impossible to transmit signals reversely. After the receiving signals are temporarily terminated, the receiving machine must be conducted with unknown balance again, as well as the signal interception and reconnaissance systems with military values. All these cannot receive training signals. Thus, the requirements on the applications reveal the importance of unknown balance technology [5].

Among various algorithms for self-adaptive unknown balance, the constant modulus algorithm (CMA) raised

\section{黑 Springer}


by Treichler is the most famous and simple one [6,7]. It utilizes the constant modulus characters of sending symbols and the high statistical amount of signal channel output. According to the single sample or multiple samples in a symbol interval, the balancer can be divided into two models: symbol interval balancer and fraction interval balancer. Therefore, the constant modulus algorithm is also divided into two types: symbol interval and fraction interval. The fraction interval balancer can reduce the sensitivity of timing phase. In the 1990s in the twentieth century, the fraction interval balancer of CMA has been used on the unknown channel balance of actual systems, such as digital HDTV system, short-code DS-CDMA system, wireless GMS cellular system, and so on [8].

However, for a balancer with limited parameters, the constant modulus algorithm sometimes would fall into an unacceptable local minimum point, and the convergent speed is very slow. For QAM-16 signal, SNR $=20$ to $35 \mathrm{~dB}$, to achieve convergence, it may require 20,000 to 30,000 data symbols. To improve the performance of CMA, this study raised an improved constant modulus algorithm. Based on the reserved original cost function, error functions and corrective terms of learning rates are added so that the algorithm can quickly find the convergent directions $[9,10]$.

For the equalizer of the present study, the tap interval is the reciprocal of the symbol rate, i.e., the symbol interval; if the equalizer before the matched filter transmits pulse after a channel distortion, then this tap interval is optimal. When the channel characteristic is unknown, the receiver filter is typically matched to the transmission signal pulses; this method leads to the equalizer performance being very sensitive to the choice of the sampling time.

\section{Conditions of signal channel balance}

Reconsider the digital communication basic band model, as shown in Figure 1. It consists of communication signal channel $h(k)$ and unknown balancer $w(k)$ connected in cascade. The mission of signal balance is to adjust each tap parameter $w_{p}(k)$ so that the ideal estimation of signal source sequence $s(k)$ can be obtained. In reality, some assumptions must be made for signal source $s(k)$, signal channel $H(z)$, and noise $n(k)$. The basic conditions are as follows $[11,12]$ :
1. The different sampled points of signal resources between $s(k)$ and $s(l)(l \neq k)$ are statistically independent.

2. Signal channel, $H(z)$, is reversible; that is, $w_{p}(k)$ exists to make balancer output $y(k) \approx c s(k-\Delta)$. Here, $c$ and $\Delta$ are any constant and integer, where $\Delta$ is time delay.

3. Noise, $n(k)$, is the Gaussian additive noise of zero mean and irrelevant to signal source $s(k)$.

The traditional balancer is based on the training sequence $d(k)$ that both sending and receiving know to adjust $w_{p}(k)$ to complete signal channel balance. The unknown balance is popular because it does not require signal channel input during working [13].

\section{Improved constant modulus algorithm}

The digital regulative communication signals have the feature of constant modulus. This feature has been widely applied on many communication applications of self-adaptive algorithm for recovery of unknown signals. The constant modulus algorithm raised by Treichler is the most famous and simple balance algorithm. It utilizes the transmitted symbol constant modulus characters and high-order statistic amount of signal channel output [14].

The constant modulus cost function is

$$
L_{\mathrm{CMA}}(y(k))=\left.\left.\frac{1}{4}|y(k)-| A_{2}\right|^{2}\right|^{2},
$$

where $A_{2}=\frac{E\left[|s(n)|^{4}\right]}{E\left[|s(n)|^{2}\right]}$ is a constant real number depending on the high-order statistic amount, whose self-adaptive algorithm can be described as

$$
\begin{aligned}
& w_{p}(k+1)=w_{p}(k)-\mu \frac{\partial L_{\mathrm{CMA}}(y(k))}{\partial w_{p}} \\
& w_{p}(k+1)=w_{p}(k)+\mu g(y(k)) x^{*}(k-p),
\end{aligned}
$$

where * is the conjugate complex, $\mu$ is the self-adaptive learning rate, $g(y)=-\partial L_{C M A}(y) / \partial y$ is the differential of the cost function depending on balancer output, socalled constant modulus error function; that is,

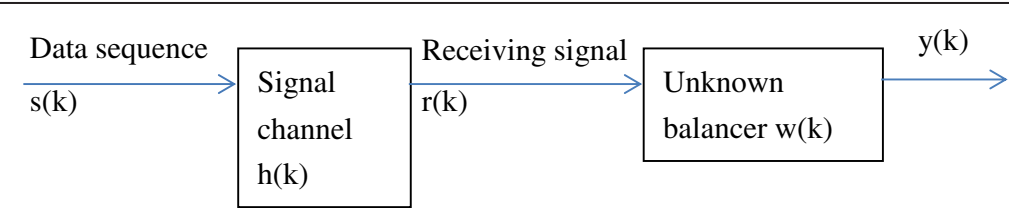

Figure 1 Unknown signal channel and balancer connected in cascade. 


$$
g(y(k))=-\frac{L_{\mathrm{CMA}}}{\partial y(k)}=\left[y(k)-\left|A_{2}\right|^{2}\right] y(k) .
$$

Thus, the traditional constant modulus algorithm confirmed by Equation 4 is

$$
\begin{aligned}
w_{p}(k+1)= & w_{p}(k)+\mu\left[A_{2}^{2}-|y(k)|^{2}\right] y(k) x \\
& *(k-p) .
\end{aligned}
$$

For a balancer with limited parameters, the constant modulus algorithm of Equation 5 sometimes would fall into an unacceptable local minimum point, and the convergent speed is slow $[15,16]$. In the self-adaptive algorithm, the convergent speed of Newton's method is faster than that of the steepest descent method because Newton's method utilizes the self-relevant messages of receiving data in the selfadaptive learning processes. Inspired by this, this study raised an improved constant modulus algorithm (ICMA), which improves the self-adaptive learning rates. The learning rates are controlled by both receiving data and balancer output. Two modifications are made on Equation 5.

The error function of Equations 1 and 4 becomes

$$
g(y(k))=(y(k)-|A|) y(k),
$$

and Equation 2 being a fixed value, learning rate $\mu$ becomes a self-adaptive learning rate. That is,

$$
\mu(k)=\mu \frac{x(k)}{y(k)} .
$$

So Equation 5 becomes

$$
\begin{aligned}
w_{p}(k+1)= & w_{p}(k)-\mu(k)(y(k)-|A|) y(k) x \\
& *(k-p) .
\end{aligned}
$$

This algorithm is called the improved constant modulus algorithm [17].

In practice, take the limited coefficients of balancer $(L+1)$, whose form is

$$
y(k)=\sum_{p=0}^{L} w_{p}(k) x(k-p)=w^{T}(k) x(k),
$$

where $w^{T}(k)=\left[w_{0}(k) w_{1}(k) \cdots w_{L}(k)\right]$ is the vector of weight coefficient of the self-adaptive signal channel unknown balancer.

$x(k)=[x(k) x(k-1) \cdots x(k-L)]^{T}$ is the status vector of the receiving sequence. Thus, the vector form of the improved constant modulus algorithm is

$$
\begin{aligned}
w(k+1)= & w(k)+\mu(k)(A-|y(k)|) y(k) x \\
& *(k),
\end{aligned}
$$

where $A=\frac{E\left[|s(n)|^{2}\right]}{E\left[|s(n)|^{1}\right]}$ is the constant real number confirmed by signal source. $\mu(k)=\mu \frac{x(k)}{y(k)}$ is the self-adaptive learning rate controlled by both receiving data and balancer output $[18,19]$.

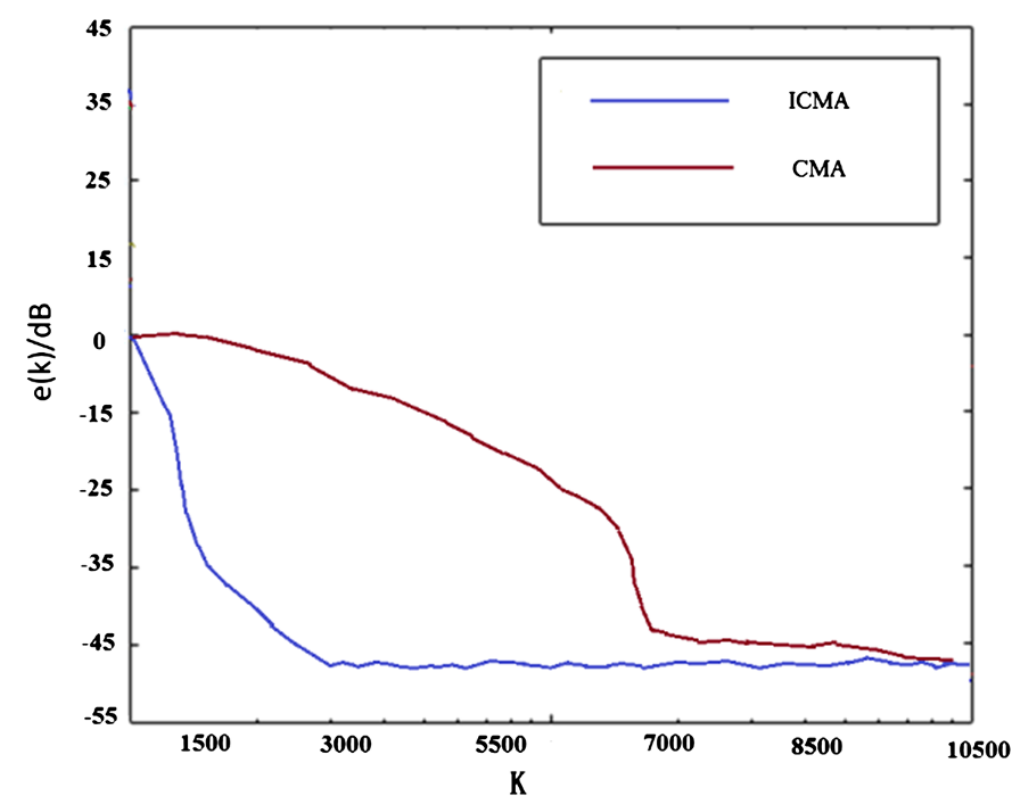

Figure 2 Comparison to interference performance among unknown balance symbol with both algorithms. 
Table 1 Comparison between improved constant modulus algorithm and traditional constant modulus algorithm at different signal/noise ratios

\begin{tabular}{|c|c|c|c|c|c|c|}
\hline \multirow{2}{*}{$\begin{array}{c}\text { Signal/ } \\
\text { noise } \\
\text { ratio } \\
\text { (dB) }\end{array}$} & \multicolumn{3}{|c|}{ ICMA } & \multicolumn{3}{|c|}{ Traditional CMA } \\
\hline & $\begin{array}{c}\text { Convergent } \\
\text { iteration time } \\
\text { (approximate) }\end{array}$ & $\begin{array}{c}\text { After } \\
\text { convergence } \\
\text { (ISI/dB) }\end{array}$ & $\begin{array}{c}\text { Balancer } \\
\text { delay }\end{array}$ & $\begin{array}{l}\text { Convergent } \\
\text { iteration time } \\
\text { (approximate) }\end{array}$ & $\begin{array}{c}\text { After } \\
\text { convergence } \\
\text { (ISI/dB) }\end{array}$ & $\begin{array}{c}\text { Balancer } \\
\text { delay }\end{array}$ \\
\hline 61 & 5,200 & -75 & 0 & 9,100 & -55 & 2 \\
\hline 55 & 5,000 & -72 & 0 & 8,800 & -52 & 3 \\
\hline 42 & 4,300 & -61 & 1 & 6,900 & -51 & 9 \\
\hline 31 & 2,800 & -56 & 2 & 8,300 & -43 & 4 \\
\hline 23 & 2,450 & -51 & 2 & 6,400 & -38 & 9 \\
\hline 17 & 2,200 & -47 & 2 & 5,600 & -36 & 14 \\
\hline 13 & 2,100 & -31 & 3 & 2,800 & -25 & 27 \\
\hline 8 & 1,300 & -26 & 1 & 5,600 & -21 & 23 \\
\hline 4 & 1,400 & -24 & 0 & 5,300 & -23 & 24 \\
\hline
\end{tabular}

\section{Experimental analysis and performance comparison}

Assume that the impulse response of transmitting real signal channel is $h=[2,0.4,-0.4,0.2,-0.2]$. Signal $s(k)$ is a binary sequence of independent distribution $\{ \pm 1\}$. Totally it has $N=10,000$ data points. The limited long balancer coefficient tap is $L=32, A=1, \mu=0.005$. The initial value of status vector $x(0)$ is evenly distributed in section [-0.2, 0.2]. To compare the traditional constant modulus algorithm of Equation 5 and the improved constant modulus algorithm of Equation 10 for unknown balance performance, it is evaluated with the interferences among residual symbol output by the balancer. The calculation formula is

$$
e(k)=\frac{\sum_{l=0}^{L}\left|c_{l}(k)\right|^{2}}{\max _{0 \leq j \leq L}\left|c_{j}(k)\right|^{2}}-1,
$$

where $c_{j}(k)$ is the convolution of balancer parameter, $w_{j}$ $(k)$, and signal channel parameter, $a_{j}[20]$.
Figure 2 shows that the interferences among residual signals are close to the same value, about $-50 \mathrm{~dB}$, when both algorithms are steady. However, at the same initial condition, the traditional constant modulus algorithm converges after 7,000 iterations while ICMA converges after 3,000 iterations, and it can quickly find the convergent direction [21].

Table 1 is the performance comparison between the improved constant modulus algorithm and the traditional constant modulus algorithm at different signal/ noise ratios $(L=64)$. Each condition is the average result of random experiments for 32 times. Through the comparison, we found that the convergent speed of the improved constant modulus algorithm is faster than that of the traditional constant modulus algorithm by 1.2 times. No delay output is found with the improved constant modulus algorithm [22].

To further examine the unknown balance ability of the algorithm with simulative experiments, the next example is the typical signal, quadrature amplitude modulation (4QAM), in the digital communication. The signal source $s(k)$ has four possible values of independent common distribution $\{ \pm 1, \pm j\}$, totally $N=10,000$ data points.
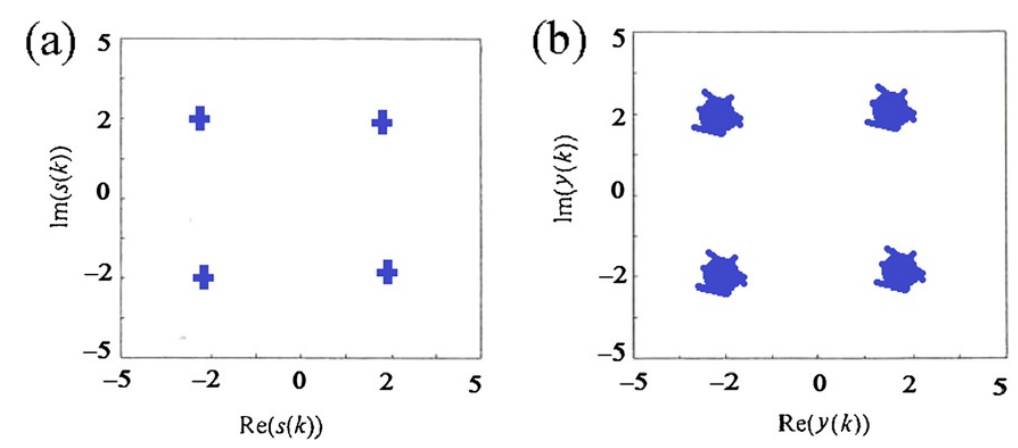

Figure 3 4QAM signal unknown balance simulative experiment diagrams. (a) Signal source. (b) Balancer output (algorithm converged). 


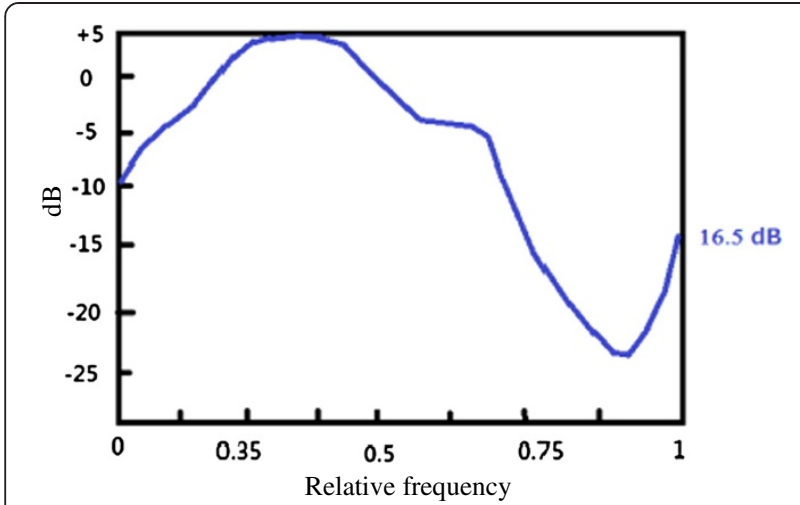

Figure 4 Amplitude spectrum of signal channel.

The impulse response of the channel is still $h=[2,0.4$, $-0.4,0.2,-0.2]$. The limited long coefficient tap $L=32$. The constant modulus constant $A=\sqrt{2}$, and $\mu=0.004$ is the initial value of status vector $x(0)$ that is evenly distributed in section $[-0.2,0.2]$. Figure 3 a shows the locations of signal source $s(k)$ on the complex plane [23].

Receiving sequence $x(k)$ is generated by the model of Equation 5. Noise is the random Gaussian noise whose zero mean square is 0.2 . Because of the influence of signal channel and noise, the signal source is seriously damaged. Figure $3 \mathrm{~b}$ is the output results of the balancer after convergence.

For $4 \mathrm{QAM}$, at the condition that $\mathrm{S} / \mathrm{N}$ ratio is equal to $20 \mathrm{~dB}$, Figure 2 shows the diagram outputs of traditional CMA and improved CMA as well as the performance curves correspondent to the interferences among symbols. It is obvious that the convergent speed of improved CMA is faster than that of traditional CMA. From the diagram output of traditional CMA (Figure 2), we can see two curves at the complex planes. This means that there are delays of balancer output. In addition, we also found that there are phase rotations in the constellation. This means that the traditional constant modulus algorithm only considers the minimum error margin, instead of phase distortion. Yuksekkaya et al. [24] raised an improved constant modulus algorithm against this. However, the algorithm is too complicate. From the constellation diagram output by improved CMA, the outputs of the balancer have neither delay nor phase rotation. The results are ideal.

The above simulation experiment shows that, compared to traditional CMA, the improved CMA raised in this study is better in terms of the unknown balance performance.

\section{ICMA frequency-selective signal channel unknown balance performance analysis}

In this simulative experiment, the signal channels are assumed as frequency-selective signal channels with three paths. The span length with seven symbols $t \in[0,5 T]$ expressed in continuous time is

$$
\begin{aligned}
h_{c}(t)= & r_{c}(t-0.25 T, \beta) \\
& +0.4 e^{-j 2 \pi(0.6)} r_{c}(t-T, \beta) \\
& -0.2 e^{-j 2 \pi(0.3)} r_{c}(t-1.5 T, \beta),
\end{aligned}
$$

where $T$ is the symbol length and $r_{c}(t, \beta)$ is the roll-off factor, raised cosine function with $\beta=0.46$. If signal channels are sampled with symbol rate, the equivalent discrete signal channel is $h_{T}(n)=h_{c}(n T)$, where $n=0,1,2$, 3. Here, the selected symbol length $T=0.008 \mathrm{~s}$. Nyquist sampling rate is $10^{6} \mathrm{~Hz}$. There are 35 sample points in the symbol length as the impulse response of signal channel is

$$
\begin{aligned}
h_{p} & =[0.6730-j 0.0217,-0.0683+j 0.3846,-0.0203 \\
& +j 0.2137,0.0231-j 0.0524] .
\end{aligned}
$$

Figure 4 shows the feature of the amplitude spectrum character of that signal channel [22].

\subsection{Interference of learning rate to error and convergent time}

Assume that the signal source is $4 \mathrm{QAM}$. The data length is 25,000 . The length of the balancer is 12 , which is equal to the length of signal channel. With the improved CMA, it is studied that when the learning rate is from (a)

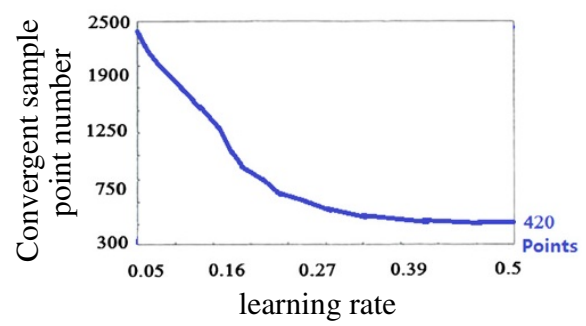

(b)

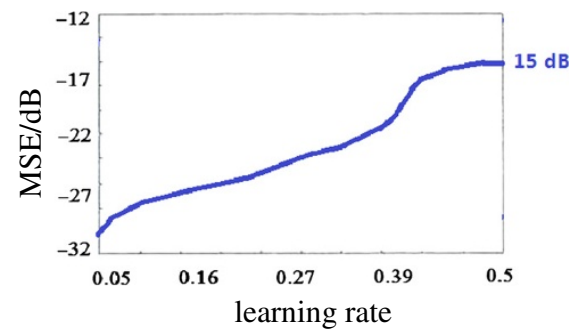

Figure $\mathbf{5}$ Influence of learning rate to balancer. (a) Influence of learning rate to convergent time. (b) Influence of learning rate to balancer performance. 
(a)

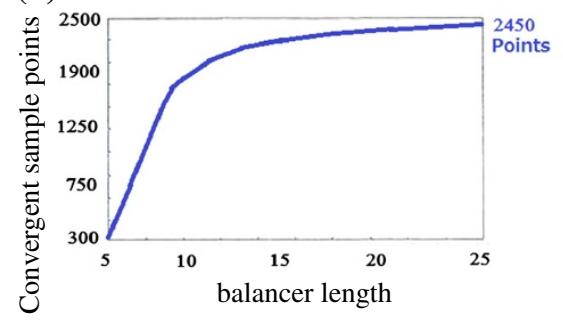

(b)

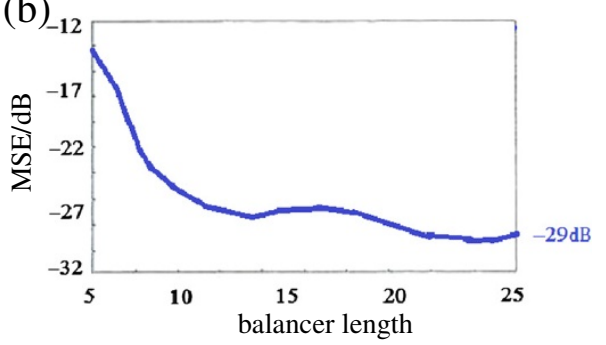

Figure $\mathbf{6}$ Influence of balancer length to balancer performance. (a) Influence of balancer length to convergent time. (b) Influence of balancer length to balancer performance.

0.05 to 0.5 , in Monte Carlo run for 43 times, the algorithm takes average of influence for convergent time and steady mean square error, as shown in Figure 5.

From Figure 5, the learning rate of ICMA influences the steady mean square error only a little, varying within the range of about $2 \mathrm{~dB}$. The learning rate obviously influences convergent time and steady residual code. For small learning rate $(0.04)$, the convergent time is about 2,500 iterations. The inter-symbol interference calculated with Equation 11 is about $-45 \mathrm{~dB}$. When the learning rate increases to 0.3 , the algorithm can converge after 450 iterations; however, the inter-symbol interference increases to about $-36 \mathrm{~dB}$.

\subsection{Influence of balance length to error and convergent time}

Assume that the signal source is 4QAM. The discrete signal channel is expressed through Equation 13. The learning rate is $\mu=0.05$. The data length is 10,000 . The balancer length is increased from 5 to 25 . The algorithm takes average in GA run for 40 times for the influences to convergent time and steady mean square error interference (see Figure 6).

From Figure 6, when the balancer length is smaller, the influences to convergent time, steady residual intersymbol interference, and mean square error are obvious, and the performances are worse. However, when the balancer length is larger than 10, these parameters are gradually stabilized. This explains that the improved CMA is not demanding to the balancer length. The advantage is that the algorithm converges fast. As for CMA and a finite impulse response (FIR) signal channel, a balancer is required for infinitive impulse response. When the balancer length is $L=32$, the steady residual ISIs are approximately equal. However, the convergent time of traditional CMA is longer [25].

\subsection{Performance comparison between ICMA and CMA}

This simulation experiment is to compare the frequency-selective signal channel unknown balance performance between ICMA and traditional CMA. Assume that the signal source is 4QAM. The discrete signal channel is expressed with Equation 13. The learning rate is $\mu=0.2$. The data length is 3,500 . The balancer length is 6 , which is equal to the signal channel length. The algorithm takes average in Monte Carlo run for 20 times. The obtained performance curve for average residual inter-symbol interference is shown in Figure 7. The blue line is the performance curve of ICMA. The maroon line is the performance curve of CMA.

The convergent time of ICMA is about 300 iterations. The steady residual ISI is about $-27 \mathrm{~dB}$. The convergent time of CMA is about 400 iterations. The steady residual ISI is about $-13 \mathrm{~dB}$. Obviously, ICMA improves the performance of the balancer in terms of convergent speed and reducing inter-symbol interference. When the balancer length is longer, the steady residual ISIs of both algorithms are approximately equal. However, the convergent speed of ICMA is still faster.

With the above two algorithms for further experiments, it was found that ICMA has better performance than CMA in terms of non-constant modulus 16QAM signals.

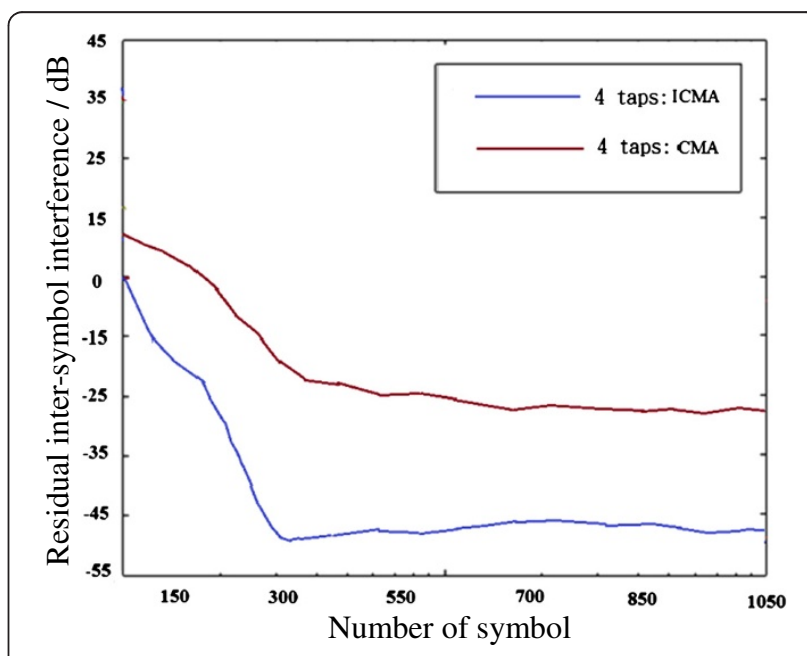

Figure 7 Average residual ISI of balancer. 


\section{Conclusion and future works}

In recent years, due to the rapid development of signal processing technologies for wireless sensing network, the signal processing that regulates the balance and estimation of unknown signal channel has become a very important technology to enhance the link. The methods and technologies applied for signal processing study gradually receive attentions and demonstrate their vigorous developmental potentials. Thus, in recent years, many universities started studying in the technical territories relevant to wireless sensing network and the internet of things, such as Central University, Feng Chia University, and Southern Taiwan University of Technology, where the courses or research centers relevant to internet of things have been established in the information technology institute to train the professional studying talents for wireless sensing network, embedded systems, and RFID technologies.

This study wishes to have new contributions and breakthroughs in the signal processing of balance and estimation for regulating unknown signal channel. This study raised the balance and estimation of regulating unknown signal channel with ICMA. This method for signal processing can be applied on various wireless sensing networks and the relay of internet of things.

From the comparison analysis and performance exploration of experimental results, we can obtain that (1) the convergent speed of ICMA is faster than that of traditional CMA, (2) the influence of learning rate to steady mean square error is smaller, varying about within the range of $1 \mathrm{~dB}$, (3) ICMA is not very demanding to balancer length, and (4) ICMA improves balancer performance in terms of convergent speed and reducing inter-symbol interference. We apply the solutions raised by this study to the technologies relevant to internet of things and develop innovations and revolutionary technologies of crossing territories to continuously improve the communication quality and performances of signal processing for wireless sensing network.

If the sampling interval is the symbol interval scores of times, such equalizer is known as the fractionally spaced equalizer, and one of the advantages of the fractionally spaced equalizer is that it is able to reduce the sensitivity of selection of sampling time. Further, for the purposes of the channel, the symbol interval equalizer and a FIR equalizer must have infinite impulse response. However, the fractionally spaced equalizer exceeds or reaches as long as the length of the channel response can be. Compared to the fractionally spaced equalizer in the 1990s, the proposed ICMA will be the next best communications technology solution to wireless sensor networking system and strategy.

\section{Competing interests}

The author declares that he has no competing interests.

\section{Acknowledgments}

This research was supported by the National Science Council of Taiwan under grants NSC 99-2623-E-167-002-ET and NSC 100-2218-E-167-001-. The authors would like to thank the National Chin-Yi University of Technology, Taiwan, for financially supporting this research.

Received: 25 November 2012 Accepted: 26 February 2013

Published: 15 April 2013

\section{References}

1. KM Passino, Biomimicry of bacterial foraging for distributed optimization and control. IEEE Control Syst Mag 22(3), 52-67 (2002)

2. S Appadwedula, W Veeravalli, DL Jones, Energy efficient detection in sensor networks. IEEE J Sel Area Comm 23(4), 693-702 (2005)

3. BG Jagyasi, BK Dey, SN Merchant, UB Desai, An MMSE based weighted aggregation scheme for event detection using wireless sensor network, in 14th European Signal Processing Conference, EUSIPCO (Florence, 2006). 4-8 Sep 2006

4. BG Jagyasi, BK Dey, SN Merchant, UB Desai, Weighted aggregation scheme with lifetime-accuracy tradeoff in wireless sensor network, in Proceedings of the 4th International Conference on Intelligent Sensing and Information Processing 2006, ICISIP (Bangalore, 2006). 15 Oct-18 Dec 2006

5. Y del Valle, GK Venayagamoorthy, S Mohagheghi, JC Hernandez, R Harley, Particle swarm optimization: basic concepts, variants and applications in power systems. IEEE Trans Evol Comput 12(2), 171-195 (2008)

6. R Blum, S Kassam, HV Poor, Distributed detection with multiple sensors: part ii - advanced topics. Proceedings of the IEEE 85(1), 64-79 (1997)

7. W Heinzelmal, A Chandrakasam, H Balakrishnan, An application-specific protocol architecture for wireless micro sensor networks. IEEE Trans Wirel Commun 1(4), 660-670 (2002)

8. Z Chair, PK Varshney, Optimal data fusion in multiple sensor detection systems. IEEE Trans Aerosp Electro Syst AES-22, 98-101 (1986)

9. Z Chair, PK Varshney, Distributed Bayesian hypothesis testing with distributed data fusion. IEEE Trans Syst Man Cybern B Cybern 18(5), 695-699 (1988)

10. B Chen, R Jiang, T Kasetkasam, PK Varshney, Channel aware decision fusion in wireless sensor networks. IEEE Trans Signal Process 52(12), 3454-3458 (2004)

11. J-J Xiao, S Cui, Z-Q Luo, AJ Goldsmith, Joint estimation in sensor networks under energy constraints, in Proceedings of the First Annual IEEE Communications Society Conference on Sensor and Ad Hoc Communications and Networks, 2004 (IEEE SECON 2004) (Santa Clara). 4-7 Oct 2004

12. IF Akyildiz, W Su, Y Sankarasubramaniam, E Cayirci, A survey on sensor networks. IEEE Communications Magazine 40(8), 102-114 (2002)

13. S Wen-Tsai, Employed BPN to multi-sensors data fusion for environment monitoring services, in Autonomic and Trusted Computing, ed. by J González Nieto, W Reif, G Wang, J Indulska,pp. 149-163. Lecture Notes in Computer Science, vol. 5586 (Springer, Berlin Heidelberg, 2009)

14. S Wen-Tsai, C Hung-Yuan, Design an Innovative Localization Engines into WSN via ZigBee and SO, in 2008 CACS International Automatic Control Conference (Tainan). 14-22 Nov 2008

15. S Wen-Tsai, Determine global energy minimum solution via Lyapunov stability theorem. IJICIC 5(7), 2011-2030 (2009)

16. A Boukerche, HAB Oliveira, EF Nakamura, AAF Loureiro, Localization systems for wireless sensor networks. IEEE Wireless Commun Mag 14(6), 6-12 (2007)

17. K Romer, F Mattern, The design space of wireless sensor networks. IEEE Trans Wireless Commun 11(6), 54-61 (2004)

18. T Wimalajeewa, SK Jayaweera, Optimal power scheduling for correlated data fusion in wireless sensor networks via constrained PSO. IEEE Trans. Wireless Commun. 7(9), 3608-3618 (2008)

19. D Nicules, B Nath, Ad hoc positioning system (APS) using AoA, in Proceedings of the IEEE INFOCOM (San FrancisCo, 2003). 30 Mar-3 Apr, pp. 1734-1743

20. LA Klein, A Boolean algebra approach to multiple sensor voting fusion. IEEE Trans Aerosp Electron Syst 29(2), 317-327 (1993)

21. T Sun, LJ Chen, CC Han, M Gerla, Reliable sensor networks for planet exploration, in Proceedings of the IEEE International Conference on Networking, Sensing and Control (Tucson, 2005). 19-22 Mar, pp. 816-821 
22. L Chia-Hung, B Ying-Wen, L Ming-Bo, Remote-controllable power outlet system for home power management. IEEE Trans Consum Electron 53(4), 1634-1641 (2007)

23. H Erdem, A Üner, A multi-channel remote controller for home and office appliances. IEEE Trans Consum Electron 55(4), 2184-2189 (2009)

24. B Yuksekkaya, AA Kayalar, MB Tosun, MK Ozcan, AZ Alkar, A GSM, internet and speech controlled wireless interactive home automation system. IEEE Trans Consum Electron 52(3), 837-843 (2006)

25. S Vernon, SS Joshi, Brain-muscle-computer interface: mobile-phone prototype development and testing. IEEE Trans Inf Technol Biomed 15(4), 531-538 (2011)

doi:10.1186/1687-1499-2013-101

Cite this article as: Sung: Wireless sensing network transmission system with improved constant modulus algorithm. EURASIP Journal on Wireless Communications and Networking 2013 2013:101.

\section{Submit your manuscript to a SpringerOpen ${ }^{\circ}$} journal and benefit from:

- Convenient online submission

- Rigorous peer review

- Immediate publication on acceptance

- Open access: articles freely available online

- High visibility within the field

- Retaining the copyright to your article 